\title{
INCEFA-PLUS (INCREASING SAFETY IN NUCLEAR POWER PLANTS BY COVERING GAPS IN ENVIRONMENTAL FATIGUE ASSESSMENT)
}

\author{
K. Mottershead ${ }^{1}$, M. Bruchhausen ${ }^{2}$, T. Métais ${ }^{3}$, S. Cicero ${ }^{4}$, I. Procopio ${ }^{4 *}$ \\ ${ }^{1}$ Amec Foster Wheeler, Walton House, Birchwood Park, Birchwood, Warrington, Cheshire, UK WA3 6GA \\ ${ }^{2}$ European Comission (DG Joint Research Centre - JRC), Westerduinweg 3, 1755 LE Petten, The Netherlands \\ ${ }^{3} \mathrm{EDF}$ (Électricité de France), 12-14 Avenue Antoine Dutrièvoz, 69628 Villeurbanne, France \\ ${ }^{4}$ LADICIM (Laboratorio de la División de Ciencia e Ingeniería de los Materiales), Universidad de Cantabria. E.T.S. de \\ Ingenieros de Caminos, Canales y Puertos, Av. Los Castros 44, 39005 Santander, España \\ * Persona de contacto: pessoai@ unican.es
}

\begin{abstract}
RESUMEN
INCEFA-PLUS es un proyecto de 5 años financiado por el programa "European Commission HORIZON2020". Iniciado en 2015, del proyecto forman parte dieciséis organizaciones de toda Europa que han aunado esfuerzos para obtener y compartir nuevos datos experimentales con el objetivo último de desarrollar una metodología de análisis del daño por fatiga ambiental en las centrales nucleares.

Algunas de estas organizaciones europeas llevaron a cabo un estudio previo al proyecto INCEFA-PLUS para desarrollar el estado del arte sobre esta temática. En este estudio se identificó, además de la amplitud de tensión/deformación, tres variables experimentales adicionales que requieren un estudio más exhaustivo con el fin de respaldar metodologías más precisas para la evaluación de la fatiga ambiental. Estas variables son: el efecto de la tensión/deformación media, períodos con tensión/deformación constante y el acabado superficial. El proyecto INCEFAPLUS estudiará experimentalmente el efecto de estas tres variables en la resistencia a fatiga de aceros inoxidables austeníticos en ambientes de reactores de agua ligera. Los datos obtenidos serán registrados y estandarizados en una base de datos online de fatiga ambiental. Un grupo de trabajo del CEN (Comité Europeo de Normalización) se dedicará exclusivamente a establecer un formato estándar de presentación de datos. Esto facilitará el intercambio de los mismos tanto dentro del proyecto como fuera de él.

Basado en los datos obtenidos y en la consecuente mejora en el entendimiento de los mismos, INCEFA-PLUS desarrollará y difundirá metodologías para incluir los nuevos datos en los procedimientos de evaluación de degradación por fatiga ambiental. Así, se tendrán en cuenta de manera más precisa los efectos de la tensión/deformación media, de los períodos con tensión/deformación constantes y del acabado superficial. Este artículo describe los antecedentes del proyecto y explica sus expectativas
\end{abstract}

PALABRAS CLAVE: Fatiga Ambiental, INCEFA-PLUS, Reactores de agua ligera, Centrales nucleares.

\begin{abstract}
INCEFA-PLUS is a major new five-year project supported by the European Commission HORIZON2020 program. The project commenced in mid-2015. Sixteen (16) organizations from across Europe have combined forces to deliver new experimental data which will support the development of improved guidelines for assessment of environmental fatigue damage to ensure safe operation of nuclear power plants.

Prior to the start of INCEFA-PLUS, an in-kind study was undertaken by several European organizations with the aim of developing the current state of the art for this technical area. In addition to stress/strain amplitude, this study identified three additional experimental variables, which required further study in order to support improved assessment methodology for environmental fatigue, namely the effects of mean stress/strain, hold time and surface finish. Within INCEFA-PLUS, the effects of these three variables on fatigue endurance of austenitic stainless steels in light water reactor environments are therefore being studied experimentally. The data obtained will be collected and standardized in an online environmental fatigue database. A dedicated CEN workshop will deliver a harmonized data format facilitating the exchange of data within the project but also beyond.

Based on the data generated and the resulting improvement in understanding, it is planned that INCEFA-PLUS will develop and disseminate methods for including the new data into assessment procedures for environmental fatigue degradation. This will take better account of the effects of mean stress/strain, hold time and surface finish. This paper will describe the background to the project and will explain the expectations for it.
\end{abstract}

KEYWORDS: Environmental Fatigue, INCEFA-PLUS, light water reactor, NPPs. 


\section{INTRODUCCIÓN}

El objetivo global del proyecto INCEFA-PLUS es desarrollar nuevas directrices para el análisis de la susceptibilidad de componentes de centrales nucleares al daño por fatiga ambiental. El proyecto está dividido en tres partes principales: la primera está enfocada en la caracterización y ensayo de una selección limitada de aleaciones de acero inoxidable austeníticos empleados en las centrales nucleares (principalmente austeníticos de bajo carbono, pero también aceros inoxidables estabilizados con $\mathrm{Ti}$ y $\mathrm{Ni}$ ); la segunda parte del proyecto implica en el desarrollo de un procedimiento modificado para la estimación la degradación de los materiales por fatiga; la última parte se refiere a la diseminación.

Este artículo introducirá el proyecto, cómo está organizado y sus expectativas. Se espera que este artículo sea regularmente actualizado, durante toda la duración de este proyecto.

\section{NOMENCLATURA}

$\begin{array}{ll}\text { AMEC } & \text { Amec Foster Wheeler } \\ \text { ANL } & \text { Argonne National Laboratory } \\ \text { CEA } & \text { Commissariat à l'Energie Atomique et aux } \\ & \text { Energies Alternatives } \\ \text { CEN } & \text { European Committee for Standardization } \\ \text { CIEMAT } & \text { Centro de Investigaciones Energéticas, } \\ & \text { Medioambientales y Tecnológicas } \\ \text { DOI } & \text { Digital Object Identifier } \\ \text { EC } & \text { European Commission } \\ \text { EDF } & \text { Electricité de France } \\ \text { EKK } & \text { E.ON Kern Kraft } \\ \text { EPRI } & \text { Electric Power Research Institute } \\ \text { IRSN } & \text { Institut de Radioprotection et de Sûreté } \\ & \text { Nucléaire } \\ \text { JRC } & \text { European Commission, Joint Research Centre } \\ \text { LEI } & \text { Lithuanian Energy Institute } \\ \text { LWR } & \text { Light Water Reactor } \\ \text { NPP } & \text { Nuclear Power Plant } \\ \text { NUGENIA } & \text { NUclear GENeration II and III Association } \\ \text { PSI } & \text { Paul Scherrer Institute } \\ \text { UC } & \text { Universidad de Cantabria } \\ \text { USNRC } & \text { US Nuclear Regulatory Commission } \\ \text { VTT } & \text { Teknologian tutkimuskeskus VTT } \\ \text { WP } & \text { Work Package } \\ \text { XML } & \text { Extensible Markup Language (computer } \\ & \text { readable document encoding rules) } \\ & \end{array}$

\section{ANTECEDENTES DEL PROYECTO}

En los últimos años se ha realizado un importante esfuerzo de investigación para abordar la influencia en la fatiga de ambientes refrigerantes a alta temperatura de los reactores de agua ligera. La Guía Reguladora 1.207 de USNRC (Comisión Reguladora Nuclear de Estados
Unidos) trata de la influencia del ambiente de reactores de agua ligera en la vida a fatiga de los materiales del reactor junto con un informe de apoyo de ANL (Laboratorio Nacional Argonne), NUREG/CR-6909 [2] en el que se presentan fórmulas para la predicción de resistencia a fatiga de aceros ferríticos y austeníticos, así como aleaciones a base de níquel, en aire y en ambiente (agua) a alta temperatura. Está claro que el efecto de los ambientes refrigerantes del reactor de agua ligera puede ser el de reducir significativamente la resistencia a fatiga en comparación con lo que se esperaría al aire. También está claro que la adopción de las fórmulas presentadas en el informe NUREG/CR-6909 requiere un cambio significativo en el enfoque para las evaluaciones de seguridad de la planta, especialmente al tener en cuenta las velocidades de deformación de los transitorios de la planta. La aplicación de los procedimientos revisados puede resultar en predicciones de factores de uso acumulado por fatiga muy altos para algunos transitorios y componentes de la planta, que parecen incompatibles con el rendimiento relativamente bueno de componentes de acero inoxidable en los reactores en funcionamiento durante varias décadas. Sin embargo, a pesar de la extensa naturaleza de los datos que se han utilizado para derivar las relaciones previstas en el informe NUREG/CR-6909, hay un número significativo de lagunas de conocimiento. Éstas fueron revisadas en un estudio reciente, patrocinado por EPRI [3].

Esta revisión se actualizó en un contexto europeo en 2013 por medio de un proyecto de NUGENIA, denominado INCEFA (Increasing safety in NPPs by Covering gaps in Environmental Fatigue Assessment). Los participantes de este proyecto eran:

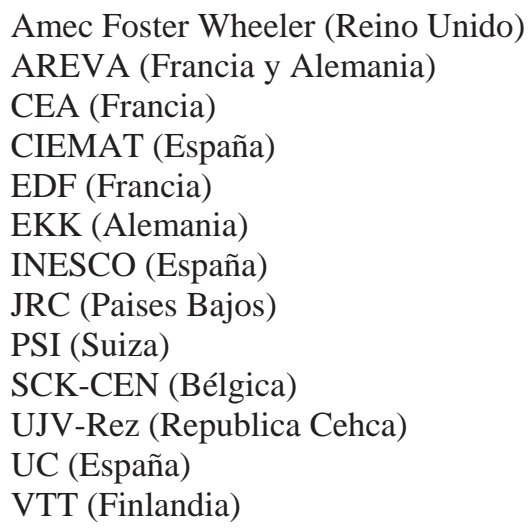

El resultado de este proyecto fue una visión europea sobre el estado del arte de la capacidad de evaluación de la fatiga asistida por ambiente, que abarca tres grandes áreas [4]:

1. Las variaciones internacionales en los procedimientos de evaluación de la fatiga asistida por ambiente y sus metas 
2. La comprensión actual de las lagunas de conocimiento que afectan la capacidad de evaluación

3. Ideas para mejorar la comprensión en beneficio de la capacidad de evaluación.

Esta revisión [4] discutió el estado actual de entendimiento sobre un número significativo de cuestiones que afectan la exactitud de la predicción de la vida a fatiga y fue capaz de identificar áreas en las cuales se consideró que un trabajo adicional era deseable. Además de la amplitud de tensión/deformación, tres de estas posibles áreas de investigación futura fueron de interés común para todos los participantes:

- Efectos de la condición superficial

- Efectos del tiempo de espera

- Efectos de la tensión/deformación media

Basado en estas conclusiones, el consorcio ha solicitado con éxito el apoyo de la Comisión Europea, en el marco del programa Horizon2020, para un proyecto de 5 años, cuyo objetivo es promover la mejor comprensión de estas tres áreas y recomendar modificaciones a los procedimientos de evaluación actuales para tener en cuenta estos efectos. El proyecto resultante fue nombrado INCEFA-PLUS y sus participantes son los mismos del consorcio original, además de:

\author{
LEI (Lituania) \\ IRSN (Francia) \\ Rolls-Royce (Reino Unido)
}

Aproximadamente el 33\% del programa es financiado por la Comisión Europea ( $€ 2.5$ millones), con el resto de la financiación proveniente de los programas nacionales de investigación.

\section{ORGANIZACIÓN DEL PROYECTO}

El Proyecto comprende cuatro "Work Packages" como se observa en la Figura 1.

En resumen, el Proyecto puede ser dividido en tres partes:

1) La primera parte centrada en los ensayos y en la campaña experimental;

2) La segunda parte se centra en analizar los resultados experimentales generados en el WP2, así como en usar otras metodologías existentes para construir una nueva metodología de fatiga (incluso curvas de fatiga y un método para fatiga asistida por ambiente) 3) La tercera parte se trata de la difusión de las lecciones aprendidas a lo largo del proyecto.
WP1: Gestión del Proyecto (AMEC)

COMPLETADO

stado del Arte (AMEC)

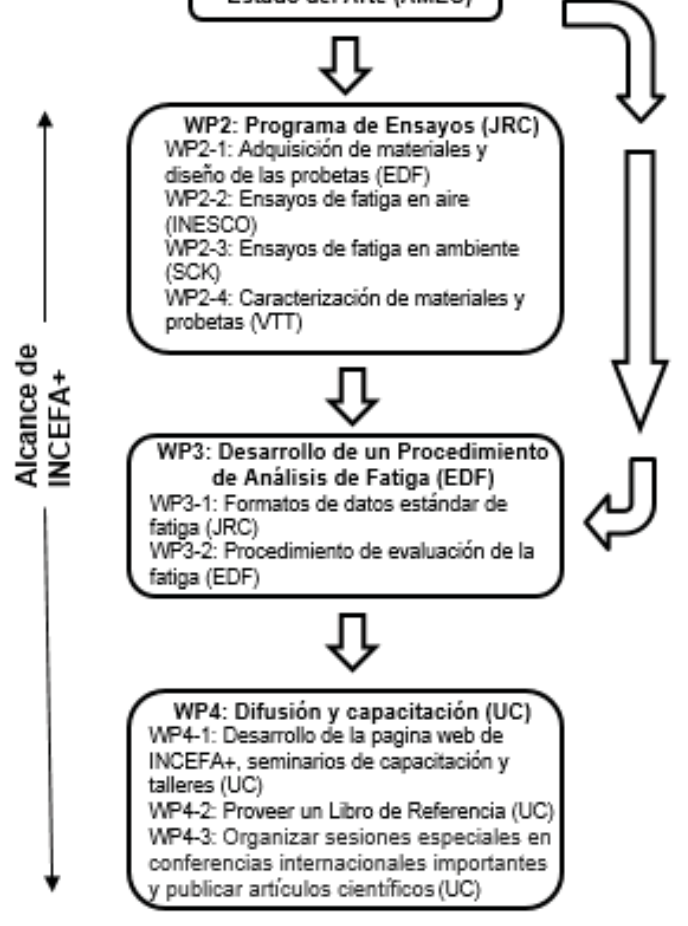

Figura 1. Diagrama de flujo organizacional del Proyecto INCEFA-PLUS

La información clave del programa incluye:

- Duración de 5 años, empezando a mediados de 2015.

- Aproximadamente 3 años de programa de ensayos, empezando a mediados de 2016.

- Un total de 13 laboratorios participantes, 11 de los cuales están comprometidos a hacer ensayos en ambiente (agua) y en aire.

- Además de los aceros inoxidables de la serie 300 de los participantes, 12 de los 13 laboratorios también harán ensayos en aceros inoxidables 304L proporcionados por EDF. Esto permite cuantificar la divergencia entre los laboratorios.

- Todos los laboratorios participantes están comprometidos a ensayar las tres variables destacadas.

- Todos los laboratorios participantes se comprometen, en la medida de lo posible, a utilizar condiciones de ensayo comunes.

- Todos los socios están comprometidos a suministrar datos en un formato común acordado.

- El proyecto apoya un CEN Workshop (del Comité Europeo de Normalización) [5] con el objetivo de desarrollar un modelo digital para implementar numéricamente los contenidos de 
la norma ISO 12106 [6]. El workshop concluye en septiembre de 2017.

- Aunque la mayoría de los ensayos se realizarán en probetas sólidas, algunos laboratorios también ensayarán probetas huecas, y uno de ellos ensayará muestras cruciformes cargadas bidireccionalmente.

- El desarrollo de programas de ensayo se aborda por medio de la metodología de Diseño de Experimentos. Este enfoque asegura que el programa aborda las interdependencias entre las variables, por lo que el efecto en la resistencia a la fatiga de una variable puede verse afectada por la variación de otra.

- Todos los socios están comprometidos con la caracterización de materiales no comunes, coherente con los datos creados para el material común.

- Se incluye un programa de caracterización de probetas con los objetivos de a) comprender los acabados superficiales obtenidos, y b) comprender las posibles razones de los resultados atípicos.

Los entregables claves que el Proyecto está comprometido a incluir son:

- Un protocolo acordado para ensayos de fatiga asistida por ambiente que el consorcio respetará, y que podría formar la base de una futura norma ISO.

- El CEN Workshop sobre formatos estandarizados de datos de fatiga y el desarrollo de una interfaz de datos XML.

- Datos de ensayos públicamente citables, individualmente identificados por DOIs (Identificadores de Objetos Digitales).

- Materiales de ensayo completamente caracterizados, complementados con una caracterización adicional del material con el fin de comprender los datos atípicos.

- Nuevas curvas de fatiga.

- Procedimientos de análisis de fatiga nuevos o modificados.

- Dos seminarios de acceso abierto.

- Un libro de referencia, adecuado para una futura conversión a una norma de evaluación.

Difusión de los resultados del proyecto mediante la participación en conferencias internacionales.

\section{ESTADO ACTUAL DEL PROYECTO Y EXPECTATIVAS A CORTO PLAZO}

El proyecto empezó a medidos de 2015. Tras el lanzamiento del proyecto, los principales logros hasta la fecha han sido:

- Acuerdo de condiciones de ensayos para la primera fase de 1 año: o Temperatura: En aire algunos ensayos pueden realizarse a temperatura ambiente para comparar los datos con la curva estándar. Sin embargo, la mayoría de los ensayos en aire y en ambiente (agua) se realizarán a $300^{\circ} \mathrm{C}$ (el rango de temperatura puede extenderse para capturar transitorios).

o Acabado superficial: Se difiere para las probetas sólidas y las huecas. Para probetas sólidas se utilizarán dos acabados, un "acabado de laboratorio pulido" $(\mathrm{Ra}=0.2 \mu \mathrm{m} ; \mathrm{Rt}=3$ $\mu \mathrm{m})$ y un "peor caso de acabado típico de la planta " $(\mathrm{Ra}=7 \mu \mathrm{m} ; \mathrm{Rt}=50 \mu \mathrm{m})$. Los orificios internos de las probetas huecas serán mecanizados usando un procedimiento común, con los valores de acabado superficial en el rango de los valores seleccionados para las probetas sólidas.

o Amplitud de deformación: Dos valores serán usados, $0.3 \%$ y $0.6 \%$.

o Tasa de deformación: Inicialmente sólo se prevé una tasa de deformación, $0.01 \% / \mathrm{s}$.

o La especificación de las condiciones fue particularmente exigente para el tiempo de espera y la tensión/deformación media; estos desafíos y sus estados actuales se describen más adelante en este artículo.

- Desarrollo del protocolo de ensayos.

- Desarrollo de especificaciones de mecanizado de probetas.

- Acuerdo de requisitos de caracterización de materiales comunes.

o Todos los socios están comprometidos a respaldar la caracterización destructiva de un ejemplar de cada probeta ensayada.

- Preparación de nuevas capacidades de ensayo para complementar las existentes.

- Comienzo de ensayos al aire y en ambiente

\section{CONSIDERACIONES SOBRE TIEMPO DE ESPERA}

Como ya se ha indicado, la elección de las condiciones de ensayo para el tiempo de espera ha sido un reto, debido tanto a los datos contradictorios en la literatura como a la necesidad de considerar las esperas relevantes para las plantas dentro de duraciones de ensayo manejables. Desde enero de 2016 muchas de las incertidumbres han sido abordadas, guiado por la experiencia de EKK y Rolls-Royce. La especificación actualmente acordada para los ensayos con tiempo de espera, incluidas las incertidumbres pendientes aún por abordar son:

- Ciclos a $300^{\circ} \mathrm{C}$

- Control de deformación

- Ensayos en aire y en ambiente. Dado que

los datos disponibles sugieren que la 
sensibilidad al ambiente para los efectos del tiempo de espera es poco probable, se considera más eficiente concentrarse en ensayos al aire. Sin embargo, también se prevén ensayos en ambiente para confirmar la sensibilidad al ambiente.

- Amplitud de deformación $=0.3 \%$ y $0.6 \%$, pero con la mayor parte de los ensayos a $0.3 \%$ Se espera que los efectos de tiempo de espera sean mayores a una amplitud de deformación baja. $0.3 \%$ se considera el valor de amplitud de deformación más alto para el cual se puede esperar un efecto significativo del tiempo de espera. Esto es en efecto un compromiso entre ensayos de largas duraciones a amplitudes de deformación bajas y los requisitos de datos para respaldar actividades de modelado posteriores.

- Tasa de deformación $=0.01 \% / \mathrm{s}$ (en aire y en ambiente)

- Temperatura de espera $=300^{\circ} \mathrm{C}$; algunos a $400^{\circ} \mathrm{C}$ (en aire)

- Tiempo de espera:

o Largas esperas (para plantas de mayor relevancia)

o 16 horas (un tiempo práctico máximo, que se confirmará mediante diálogo con los operadores de la planta)

o Posiblemente se incluyan diferentes tiempos de espera para evaluar la importancia del endurecimiento por deformación

- Frecuencia de espera

o Diferentes a amplitudes de deformación de $0.3 \%$ y $0.6 \%$ (propuesto 20.000 ciclos a $0.3 \%$; 5.000 ciclos a $0.6 \%$ )

o A confirmar mediante diálogo con el operador

- Forma de onda = onda de sierra (caída de carga más rápida para ayudar la eficiencia del ensayo)

- Deformación durante la espera = cero (las más bajas son las más relevantes para la planta)

- Posición de espera = después de la tasa de deformación positiva

\section{CONSIDERACIONES SOBRE DEFORMACIÓN MEDIA}

TENSIÓN/

Para la tensión/deformación media, los problemas que afectan a la elección de los parámetros de ensayo son particularmente complejos y el proyecto ha realizado un esfuerzo significativo para crear un programa de ensayos que sea a) práctico, y b) capaz de proporcionar información útil para las evaluaciones de plantas.
Las opciones de las condiciones de ensayo y sus características son:

- Tensión media combinada con control de deformación.

o Se está de acuerdo que este es el tipo de ensayo más relevante para los componentes (p.ej. tubería) sometidos tanto a cargas térmicas transitorias como a cargas estáticas debido a presión.

o Es un ensayo complejo de implementar. Existe cierta experiencia en este tipo de ensayos (EDF y CEA) sobre la cual podría basarse una campaña. Sin embargo, la inversión en capacidad de ensayo probablemente sería significativa.

- Tensión media combinada con control de carga.

o Ensayo viable de realizar.

o La tensión media es relevante para la planta, p.ej. para componentes como tuberías sujetas a presión.

o La experiencia de PSI hasta la fecha sugiere que se requiere fatiga de alto número de ciclos antes de que efectos significativos de tensión media sean evidentes.

- Deformación media con control de deformación.

o Ensayo viable de realizar.

o El control de deformación es más relevante para el régimen de fatiga de bajo número de ciclos (que es el enfoque principal de INCEFA).

o Los resultados de los ensayos en control de deformación son directamente comparables con los datos ya disponibles.

Otra consideración es el nivel de tensión o deformación al que se debe aplicar la tensión media o deformación media. Basado en los aportes de las contribuciones del Proyecto INCEFA, se llegó a la conclusión de que los ensayos se realizarían a bajas amplitudes tensión/deformación, es decir, en el dominio de fatiga de alto número de ciclos. Se observa que el efecto de la tensión/deformación media es muy limitado en el dominio de fatiga de bajo número de ciclos ya que la contribución de la tensión/deformación media tiende a redistribuirse a través del espesor del componente.

Basado en estas consideraciones, un programa práctico y útil ha sido desarrollado de la siguiente manera:

- La fase 1 del programa implica un número limitado de ensayos de tensión media en control de tensión y está enfocada en ensayos de deformación media en control de deformación. 
o Estos ensayos pueden ser realizados por todos los socios sin necesidad de desarrollar técnicas de ensayo.

o Los ensayos se están llevando a cabo tanto en aire como en ambiente.

o Los ensayos permitirán que los datos disponibles para estas condiciones sean cuestionados y confirmados o refinados. o Para los ensayos en control de deformación, se usará $0 \%$ y $0.5 \%$ de deformación media combinada con $0.3 \%$ o $0.6 \%$ de amplitud de deformación. La amplitud de deformación proporciona una comparación con otros datos obtenidos por el proyecto, mientras que la deformación media es aproximadamente equivalente a la tensión media relevante de la planta.

o Los parámetros para los ensayos en control de tensión serán determinados por PSI ya que éstos forman parte del programa Nacional Suizo que se aporta al proyecto.

- La fase 2 del Proyecto empezará en 2017 y comprende ensayos exploratorios usando, entre otros, tensión media en control de deformación.

o Teniendo en cuenta las probables complejidades del control de los ensayos, éstos van a empezar en ambiente al aire.

o El alcance y la dirección del programa dependerán en primer lugar del efecto de la deformación media revelada por los ensayos exploratorios, y en segundo lugar las restricciones (constraints) prácticas de ensayo.

\section{CONCLUSIONES}

Este artículo presenta el proyecto INCEFA-PLUS, sus objetivos y organización a la comunidad en general. También resume el estado actual del proyecto y sus retos. Se prevé que este artículo se actualizará durante todo el curso del proyecto para su difusión continua a la comunidad en general.

\section{AGRADECIMIENTOS}

Este proyecto ha recibido financiación del programa de investigación y formación de Euratom 2014-2018 bajo acuerdo de subvención No 662320.

También se reconocen las contribuciones significativas de todos los socios del proyecto INCEFA-PLUS.

\section{REFERENCIAS}

[1] US-NRC Regulatory Guide 1.207, "Guidelines for Evaluating Fatigue Analyses Incorporating the Life Reduction of Metal Components due to the Effects of the Light-Water Reactor Environment for New Reactors", March 2007.

[2] O.K. Chopra, W.J Shack, "Effect of LWR Coolant Environments on the Fatigue Life of Reactor Materials": U.S. Nuclear Regulatory Commission. NUREG/CR-6909 and ANL-06/08, February 2007

[3] D.R. Tice, D. Green, A. Toft, "Environmentally Assisted Fatigue Gap Analysis and Roadmap for Future Research - Gap Analysis Report”, EPRI 1023012, December 2011

[4] Environmentally-Assisted Fatigue Assessment The European view of the State of the Art for stainless steels in LWR environments. NUGENIA position paper available at www.nugenia.org.

[5] CEN Workshop on Standards Compliant Formats for fatigue Test Data - FATEDA. www.cen.eu/Pages/resultsearch.aspx?k=FATEDA.

[6] ISO 12106, Metallic materials. Fatigue testing. Axial-strain-controlled method. 